\title{
Der „,imperfekte“ Körper auf dem Heiratsmarkt - ein Beitrag zu Disability in Heiratsannoncen des Kaiserreichs
}

Tamara Frey

\begin{abstract}
„Durch die Spalten der periodischen Presse in den Hafen der ehelichen Glückseligkeit einzulaufen, ist auch bei uns in Deutschland ein nicht mehr ungewöhnlicher Weg“ - mit diesen Worten leitete die wohl berühmteste Familienzeitschrift Deutschlands im 19. Jahrhundert, die Gartenlaube, 1873 einen Artikel ein, der die Eheanbahnung durch Heiratsannoncen zum Thema hatte. ${ }^{1}$ Tatsächlich gehörten Anzeigen, in denen Männer oder Frauen einen Partner des jeweils anderen Geschlechts zum Zweck der Ehe suchten zum täglichen Bild der Inseratenseiten in großen deutschen Tageszeitungen. ${ }^{2}$ Stellengesuche, Immobilienanzeigen, Warenofferten und Angebote diverser Dienstleistungen schufen einen Marktplatz aller Art, der Kommunikation und Austausch auch außerhalb der personalen Netzwerke und zwischen räumlich getrennten Menschen ermöglichte. Durch Heiratsannoncen lokalisierte sich nun ein weiterer Markt in den Inseratenseiten, der sich durch die Verschleierung seiner ökonomischen Mechanismen charakterisiert - der
\end{abstract}

1 Art. „Die Ehezeitung“, in: Gartenlaube (1873/Heft 38), S. 613-615, hier S. 613.

2 Eine breit angelegte Durchsicht von 20 Zeitungen und Zeitschriften und sechs Untersuchungsjahren über den Zeitraum des Kaiserreichs im Rahmen der Dissertation „"Strengste Verschwiegenheit auf Manneswort!“ Eine Analyse von Heiratsannoncen im Kaiserreich“ ergab eine Gesamtzahl an Heiratsannoncen von rund 41.000 . 
Heiratsmarkt (vgl. dazu: Bourdieu: 1987: 207). Auch hier gelten die Regeln von Angebot und Nachfrage. Auf dem Heiratsmarkt präsentieren sich die beteiligten Personen als heiratsfähige Subjekte und werden als mehr oder minder „gute Partien“ eingestuft. Es findet eine „soziale Verortung“ im Sinne Pierre Bourdieus statt. Diese soziale Verortung erfolgt über die Verteilung von bestimmten Kapitalsorten, die als Ressourcen zu verstehen sind (zu den Kapitalsorten nach Bourdieu vgl.: Bourdieu 1983: 185; Manderscheidt 2008: 158; zu Kapitalsorten als Ressourcen vgl.: Schroer 2012: 98). Welche Kapitalsorten eingesetzt werden können, um in eine anerkannte Position zu gelangen, ist auf die Möglichkeit der späteren Ehe bezogen. Da Männern und Frauen im Kaiserreich in der Ehe unterschiedliche Rollen zugeschrieben wurden, verhalten sich auch die Ansprüche an sie in den Anzeigen genderspezifisch. Die Geschlechtsidentität ist damit die entscheidende soziale Rolle auf dem Heiratsmarkt. Dadurch wird die Ausgestaltung der Präsentationen innerhalb des Sets für diese soziale Rolle und der mit ihr verbundenen Verhaltenserwartungen und normativen Konzepte geprägt - ein „doing gender“ findet statt (zum Konzept des „,doing gender“ vgl.: West/ Zimmerman 1991: 13-37).

Zeitgenössische Kritik an der Eheanbahnung durch Annonce stufte das Heiratsinserat nicht nur als die Sittlichkeit bedrohenden „Hort der Unzucht“ ein (Bloch 1908: 786-791), gerade aus sozialdemokratischer Richtung wurde darauf hingewiesen, dass die Heiratsannonce den Charakter der bürgerlichen Ehe als Kaufgeschäft verrate, indem sie den „Nimbus“ abstreife, den die Dichter um sie gewoben hätten (Bernstein 1895: 17-19). Margot Berghaus' Überlegungen zu den strukturellen Eigengesetzen von Heirats- und Bekanntschaftsanzeigen können dieses Argument von politischer Färbung befreien. Sie macht deutlich, dass es der Zwang des Mediums zur sprachlichen Zieldefinition ist, der den Tauschprozess zwischen den Kapitalsorten durch eine Sprache offenlegt, die vor allem das ausdrücken kann, was mess-, quantifizier- und berechenbar ist (Berghaus 1986: 59/62f.). Dabei finden sich Kapitalsorten sowohl in der Selbstpräsentation der Inserenten als auch in der Präsentation der Beschreibungen eines Wunschpartners. Der Prozess des Kennenlernens findet bei der Eheanbahnung durch Annonce in einem einmalig medial bereitgestellten Kommunikationsraum statt, dessen Platz begrenzt ist und der durch eine zielgerichtete Sprache den für die soziale Verortung unerlässlichen Einsatz von Kapitalsorten besonders deutlich macht. 
Doch was, wenn das kapitalbasierte „doing gender“ von einer weiteren Kategorie flankiert wird, die ebenfalls als maßgebliches Referenzsystem und Ordnungsprinzip soziokulturelle und hierarchische Differenz produziert (Raab 2007: 127-148) und nach zeitgenössischer Auffassung mit Devianz, Inferiorität, Abhängigkeit und allgemeinen Defizitzuschreibungen konnotiert war (dazu: Bösl 2010: 29-43; Garland-Thomson 1997) und damit nicht als Kapitalsorte eingesetzt werden konnte, sondern im Gegenteil die soziale Einordnung zu schwächen schien? Dieser Aufsatz wird sich mit sechs Heiratsannoncen befassen, deren Inserierende in ihrer Selbstbeschreibung körperliche Charakteristiken thematisieren, die medizinisch als ,impairment“ eingeordnet werden und die in der sozialen Konstruktion als kulturelles und gesellschaftliches Differenzierungsmerkmal fungierten (zur Unterscheidung von ,impairment“ und „disability“: Waldschmidt 2010: 13-27). Dabei wird zum einen die Wechselbeziehung der Kategorie „Behinderung“ mit der Kategorie „Geschlecht“ in den Blick genommen und untersucht, wie sich die genderspezifischen Implikationen im sozialen Raum ausformten. Zum anderen wird an einem speziellen Ort, der Annonce, der sich mit einem speziellen Kontext, dem Heiratsmarkt, zu einem textlichen Kommunikationsraum verbindet, kenntlich gemacht, wie die verschiedenen Differenzmarkierungen ihre Wirkmacht entfalteten und sprachliche Handlungen prägten (zu dieser Forschungsfrage vgl.: Raab 2007: 137).

\section{MÄNNLICHKEIT UND DISABILITY}

Die in diesem Aufsatz untersuchten Heiratsannoncen wurden während der Zeit des Kaiserreichs in verschiedenen deutschen Tageszeitungen geschaltet. In vier der Anzeigen sucht eine männliche Person einen weiblichen Ehepartner, in zwei Annoncen wird ein Ehemann für eine weibliche Person gesucht. Dieses 2:1-Verhältnis spiegelt die allgemeine Geschlechterverteilung bei der Eheanbahnung durch Annonce wider. Gemäß den normativen Rollen des (männlichen) aktiven Werbers und der (weiblichen) passiven Umworbenen stellten Männer auch auf diesem speziellen Heiratsmarkt häufiger Offerten als Frauen.

Die erste Annonce, die hier vorgestellt werden soll, wurde im Juli 1898 im Berliner Tageblatt geschaltet und definiert mit der Überschrift „Avis für 
Taubstumme" bereits die Zielgruppe dieses Heiratsgesuchs. ${ }^{3}$ Die Überschrift war, im Gegensatz zum Text der Annonce, in einer serifenlosen Schrift und ca. dreimal so groß wie die Buchstaben des eigentlichen Gesuches gesetzt. Annoncen von oder für gehörlose Menschen gehörten nicht zum täglichen Bild der Anzeigenseiten der gängigen deutschen Tagesanzeigen. Es ist deshalb anzunehmen, dass mit diesem besonderen Layout die Sichtbarkeit der Anzeige verstärkt werden sollte, um die entsprechende Zielgruppe auf den ersten Blick auf diese aufmerksam zu machen. Die erste Zeile des Textes „Für meinen taubstummen Vetter" macht deutlich, dass der Suchende hier nicht selbst als Inserierender auftrat, sondern ein Verwandter oder eine Verwandte. Diese Konstellation bedeutet jedoch nicht, dass dem Suchenden hier auf Grund der Einordnung als taubstumm eine aktive Rolle im Prozess der Eheanbahnung abgesprochen wurde. Das Vorgehen über einen Mittler war im Kaiserreich nicht ungewöhnlich. Circa zehn Prozent der Inserate, in der ein Mann eine Ehepartnerin suchte, wurden nicht von ihm selbst, sondern von Verwandten, Freunden oder Vermittlern geschaltet. Bei Frauen gilt dies sogar für ein Drittel der Anzeigen. Die zweite Zeile der Anzeige setzt die Beschreibung des Vetters als „,selbstständigen Fabrikanten in Wien“ fort. Hier wird der Beruf des Suchenden genannt und zudem mit einem Adjektiv versehen, das Unabhängigkeit suggeriert. Auch die Angabe des Wohnortes ist interessant. Sie deckt sich mit der Angabe, dass Antwortbriefe an die Agentur Rudolf Mosse in Wien gesendet werden sollten. Rudolf Mosse, der Herausgeber des Berliner Tageblattes, begann seinen steilen Aufstieg in der Medienbranche durch die Etablierung eines internationalen Netzes sogenannter „Annoncenexpeditionen“, die für Privat- und Geschäftskunden Anzeigen in verschiedenen Zeitungen platzierten (zur Geschichte des Unternehmens siehe z.B.: Kraus 1999: 77-101). Die Tatsache, dass für einen in Wien ansässigen Mann in einer Berliner Zeitung nach einer Ehepartnerin gesucht wurde, lässt darauf schließen, dass es für sinnvoll erachtet wurde, die raumüberwindende Dienstleistung der Agentur Rudolf Mosse in Anspruch zu nehmen. Dies hat sicher mit der speziellen Zielgruppe der Annonce zu tun, die es erforderlich machte, den Suchradius auszudehnen. Die nächsten beiden Zeilen umfassen die Beschreibung der gewünschten Partnerin als eine „aus

3 Berliner Tageblatt und Handelszeitung vom 17.07.1898, Chiffre W.H. 2854; Die Zeitung liegt in digitalisierter Form unter http://zefys.staatsbibliothek-berlin.de /list/title/zdb/27646518/ vor (letzter Zugriff 16.02.2017). 
besserer Familie“ stammende ,taubstumme Lebensgefährtin mit Vermögen“. Zuletzt folgen wiederum einige Angaben über den Suchenden: das Alter (33 Jahre) und einen Hinweis auf seine materielle Sicherheit (,,in sehr geordneten Verhältnissen“). Zwar ist die Angabe des Tätigkeitsfeldes „Fabrikant" wenig aufschlussreich, da sie vom kleinen Hinterhofhutgewerbe bis zum großen Stahlmagnaten alles umfassen könnte, doch wird sowohl mit der Beschreibung von ,geordneten Verhältnissen“ als auch der Forderung nach einer Partnerin aus „guter Familie“ und materiellen Sicherheiten das Bild bürgerlicher Lebensverhältnisse aufgebaut.

Ähnlich verhält es sich bei der zweiten Anzeige, die hier eingeführt werden soll. Sie erschien im März 1908 ebenfalls im Berliner Tageblatt, trägt keine Überschrift, ist aber in der seltenen Ich-Formulierung verfasst. ${ }^{4}$ Der Inserent eröffnet sein Gesuch mit einer detaillierten Beschreibung seiner Wunschpartnerin. Er beginnt mit dem Alter (20-26), das sie haben sollte und fährt mit der Angabe fort, dass kein Vermögen notwendig sei. Dies wird durch Fettdruck besonders sichtbar gemacht. Durch die direkt daran anschließende Beschreibung, dass sie ,jed[och] e[ine] schöne und zu mir pass[ende] Figur besitzt, hochgebildet ist und auf literar[ischem] oder musikal[ischem] Gebiete möglichst künstler[ische] Fertigkeit erreicht hat", erscheinen die körperlichen und kulturellen Anforderungen an die Wunschpartnerin in direktem Bezug zum vorhergehenden Verzicht auf Materielles zu liegen. So entsteht der Eindruck, dass der Inserent bewusst diese Auswahlkriterien vor die materiellen setzte. Auch über diese Beschreibung entsteht das Bild einer Frau aus der bürgerlichen Gesellschaftsschicht, die, von Erwerbsarbeit freigestellt, Zeit und geistige Förderung besaß, die es ihr erlaubte, sich zu bilden. In einem zweiten Satz beschreibt sich der Inserent nun selbst. Er beginnt seine Selbstbeschreibung mit einer ausführlichen Darstellung körperlicher Eigenschaften (,1,80 m. groß, schlank, brünett, von vorteilhaftem Aeußeren") und schließt sein Alter an (Mitte 30). In einem mit einem ,und“ verbundenen zweiten Hauptsatz legt er dar, dass er ,,einen kleinen Gesichtsfehler, der aber nicht im geringsten entstellt oder beruflich hindert" habe. Eine Angabe des Jahreseinkommens (10.000) und seines Berufes (Großkaufmann) schließt sich an. Auch er setzte sich so in das Berufsspektrum des Bürgertums. Neben der expliziten Kenntlichmachung der eigenen Person durch die unübliche „Ich-Formulierung“, weist diese Annonce auch

4 Berliner Tageblatt und Handelszeitung vom 05.03.1908, Chiffre T.K. 890. 
einen überdurchschnittlichen Anteil an körperlichen Beschreibungselementen auf. Nur in einem Drittel der Anzeigen männlicher Suchender wurde der eigene Körper überhaupt thematisiert, nur jeder Fünfte beschrieb körperliche Attribute, die sich auf die Wunschpartnerin bezogen (Ergebnisse aus Frey 2017).

Während sich diese Annonce durch detaillierte Beschreibungen auszeichnet, ist die dritte Anzeige, die hier analysiert werden soll, sehr kurz gehalten. Die Überschrift „Blinder Mann“ ist dabei durch einen Relativsatz mit dem Beginn des nur vier Zeilen langen Textes verknüpft und stellt abermals die ökonomische Autonomie des Inserenten ins Zentrum (,welcher selbstständiges Geschäft hat, sucht..."). ${ }^{5}$ Die Wunschpartnerin wird nicht weiter beschrieben. Die Klientel der Münchner neueste Nachrichten war, anders als die des Berliner Tageblattes, eher kleinbürgerlich geprägt. Da die Kosten für die Annonce auf Zeilen gerechnet wurden, lässt die kurze Anzeige darauf schließen, dass nur wenig Kapital zur Verfügung stand, um die Heiratsabsicht darzulegen und sich selbst vorzustellen bzw. die Wunschpartnerin zu beschreiben.

Die vierte Anzeige unterscheidet sich von den vorherigen vor allem dadurch, dass die Heiratsabsicht nicht an erster Stelle steht, sondern erst in einem zweiten Satz als Option erscheint (,Bin auch nicht abgeneigt, zu heirathen, um für immer einen vertraulichen Menschen zu haben“). ${ }^{6}$ Während der Inserent der zweiten Anzeige das „Ich“ in seinem Inserat explizit macht, schreibt zwar auch der Inserent hier in der ersten Person, doch spart er eine explizite Selbstbenennung aus und beginnt mit dem konjugierten Verb; ein Schreibstil, der in einigen Annoncen angewendet wurde, die in der ersten Person verfasst waren. Dass anstatt der üblichen Chiffre tatsächlich ein Name mit Adresse genannt wurde, war dagegen eine Seltenheit. Zwar ist nicht zu rekonstruieren, ob es sich um den Inserenten selbst oder eine Mittelsperson handelte, doch wurde hier bewusst die Möglichkeit der Anonymität, die die Eheanbahnung durch Annonce bietet, ungenutzt gelassen. Das Hauptanliegen des Inserenten ist es, durch die Anzeige eine „ehrliche und zutrauliche" weibliche Person (ledig oder kinderlos verwitwet) zu finden, „die mich herumfährt, in Gasthäusern, mit einem Krankenwagen“. Die

5 Münchner neueste Nachrichten vom 10.04.1908, Chiffre J.W.113689.

6 Berliner Zeitung vom 08.06.1898, Willibald Jakubetz. 
knappe Selbstbeschreibung „,bin Musiker und kann schlecht laufen“ folgt darauf. Wie bei der Münchner neueste Nachrichten rangierte auch die Klientel der Berliner Zeitung im klein- und unterbürgerlichen Bereich. Auch das Tätigkeitsfeld des Inserenten fällt aus dem Spektrum der für das Bürgertum adäquat angesehenen Berufe heraus.

Wie die Bürgertumsforschung der letzten Dekaden zeigte, war das Verhältnis der kulturell führenden Schicht des 19. Jahrhunderts zu über den Körper wahrgenommenen Differenzen komplex, sei es in Bezug auf die Hautfarbe eines Menschen, einer vom biologischen Geschlecht abweichenden sexuellen Orientierung oder, wie Arbeiten aus der „Disability History“ zeigen, einer medizinisch diagnostizierten „Andersheit“. Letzteres soll an den für dieses Verhältnis maßgebenden Eckpunkten Definitionsmacht, Selbstverständnis und Abgrenzung näher untersucht und mit den oben dargestellten Inhalten der Heiratsannoncen in Bezug gesetzt werden.

Wie Lennard J. Davis in seiner Abhandlung ,Enforcing Normalcy“ herausarbeitet, führte der Aufstieg des statistischen Systems, der Daten und Messungen, zur Anwendung einer Kategorie auf den Menschen, die den Durchschnitt zur Richtschnur definierte und damit weitreichende Folgen für alle hatte, die aus irgendwelchen Gründen nicht in das evozierte Bild des „Normalen“ fielen (Davis 1997: 9-28). [MONSTER] Die Herausgeber des Sammelbandes „Der (im-)perfekte Mensch“ formulierten den so hervorgebrachten Mechanismus passend: „Erst die Erfindung der Normalität hat die Tableaus der Abweichung hervorgebracht“ (Lutz/Macho/Staupe/Zirden 2003: 11). Das „Normale“, so Davis, wurde Teil des großen Entwurfes von Fortschritt, Industrialisierung und der Stabilisierung bürgerlicher Macht (Davis 1997: 26).

Nicht nur das statistische Konzept von einer durchschnittlichen staatlichen wie privaten Wirtschaftsleistung verbindet so die Vorstellung von Norm und Abweichung mit dem Feld der Arbeit, auch das bürgerliche Selbstverständnis rückt diesen Bereich in zentrale Stellung. In ihrem Enzyklopädieartikel zur Industrialisierung weist Anne Borsay darauf hin, dass ,,impairment", unabhängig vom Geschlecht, als Mangel mit schädlichen wirtschaftlichen Folgen angesehen wurde (Borsay 2006: 947-950). Hier sollen zunächst die Implikationen für männliche Personen näher dargestellt werden; für weibliche Personen wird dieser Punkt im nächsten Unterkapitel ausgeführt. Berufstätigkeit gehörte zu einem gelungenen männlichen bürgerlichen Lebenslauf, denn durch sie war der bürgerliche Mann erst in der Lage, seine 
identitätsstiftende Rolle als (alleiniger) Ernährer der Familie zu erfüllen (vgl.: Coontz 2005: 188). Doch nicht nur im bürgerlichen Spektrum nahm der Beruf eine große Rolle für die Identitätsbildung ein. Auch in Gesellschaftsschichten unterhalb des Bürgertums wertet die Forschung die ,auf körperliche Arbeit beruhende Produktionsleistung" (Zwahr 1978: 113 nach Kaschuba 1990: 94) als Teil des Selbstverständnisses. Die Arbeit wurde dabei mit Abstraktionen wie Reife, Selbstständigkeit und daraus resultierender Ehre in Verbindung gesetzt. (Kaschuba 1990: 95). Rosemarie GarlandThomson macht die Bedeutung eines stets einsatzbereiten Körpers für das Konzept der Selbstständigkeit deutlich, mit dem sich der Einzelne einen Platz im umkämpften und dynamischen wirtschaftlichen Feld sichern könne (Garland-Thomson 1997: 43). Das große normative Konzept der Selbstständigkeit rücke die eigene Verantwortlichkeit für die soziale und ökonomische Situation ins Zentrum (Garland-Thomson 1997: 43). Das Bild des durch ein Narrativ von körperlicher Unzulänglichkeit hergestellten behinderten Menschen, bilde dabei das kulturelle Gegenstück zum Ideal des selbstbestimmten und normierten Individuums (Garland-Thomson 1997: 41).

Schauen wir uns nun noch einmal die Inhalte der oben beschriebenen Heiratsannoncen an, dann ergibt sich der Eindruck, dass die Inserenten dieser negativen Einordnung bewusst entgegenarbeiten wollten. Nicht nur wird in allen Anzeigen eine Einkommensquelle genannt (diese Angabe gehört zu den integralen Bestandteilen einer Heiratsannonce im Kaiserreich), sie wurde in den Fällen des blinden Mannes und des taubstummen Vetters zusätzlich mit dem Adjektiv „selbstständig“ versehen. Dem gängigen Defizitnarrativ des abhängigen Behinderten, der zu produktiver Erwerbstätigkeit unfähig ist (Bösl 2010: 31), der es nicht schafft, sich seinen eigenen Lebensunterhalt zu verdienen und stattdessen auf Hilfe von Familie oder Almosen angewiesen ist, wird damit deutlich widersprochen. Der Großkaufmann mit einem „Gesichtsfehler“ geht hier sogar noch weiter. Er baut in seiner Anzeige durch die selbstbewusste Ich-Formulierung, die Nennung körperlicher Vorzüge und detaillierter Forderungen an seine künftige Ehepartnerin das Bild eines attraktiven und selbstsicheren Mannes auf. Erst dann kommt er auf seine Imperfektion zu sprechen, die er jedoch sofort mit der abschwächenden Erklärung versieht, dass diese ,nicht im geringsten entstellt oder beruflich hindert“. Hier wird ein Gedankengang des Lesers, der Mangel mit schädlichen wirtschaftlichen Folgen verbindet, antizipiert, sofort unterbro- 
chen und widerlegt. Unterstützt wird diese Erklärung dann durch den folgenden Satz, in dem der Inserent einen Beruf des bürgerlichen Spektrums nennt sowie ein Jahreseinkommen, mit dem er eine Familie dem bürgerlichen Lebensstil angemessen unterhalten konnte.

Lediglich die letztgenannte Annonce des Musikers hält die Darlegung der Selbstständigkeit nicht aufrecht. Zwar wird auch hier eine Einkommensquelle genannt, doch sucht er in seiner Anzeige gezielt eine Helferin, die ihn unterstützt. Während sich vor allem die Inserenten des Berliner Tageblattes allein durch die Tatsache, aber auch die Art ihrer Erwerbstätigkeit in ein bürgerliches Spektrum setzten, scheint das Milieu des Musikers eher dem entsprochen zu haben, was Brendan Gleeson für die industrialisierte Stadt beschreibt, in der Menschen mit Behinderung zum Bild des quirligen, modernen öffentlichen Raumes gehörten, in dem sie ein gewisses Maß an wirtschaftlicher Unabhängigkeit erreichen konnten (Gleeson 1999: 110f). Der Wunsch des Inserenten ,für immer einem vertraulichen Menschen“ haben zu wollen, kann als Ausdruck für das unstete und mitunter einsame Leben am Rande der bürgerlichen Gesellschaft gewertet werden, das durch die Heirat Stabilität und anhaltende Gesellschaft erhalten sollte.

\section{WEIBLICHKEIT UND DISABILITY}

Eingangs wurde erwähnt, dass etwa zehn Prozent der Heiratsannoncen für Männer und ein Drittel der Heiratsannoncen für Frauen, nicht von den Suchenden selbst sondern von Verwandten, Freunden oder Vermittlern geschaltet wurden. Dieser Wert erhöht sich noch einmal, wenn die Personen der jüdischen Religionsgruppe angehörten. So waren zwei Drittel der Heiratsannoncen, in denen jüdische Frauen den suchenden Part einnahmen, nicht von ihnen selbst, sondern durch Mittelspersonen geschalten. Die Eheanbahnung durch Dritte nahm für die verstreut lebende jüdische Bevölkerung eine zentrale Rolle ein (Maurer 1991: 345). Ein passender Ehepartner wurde über die Verwandtschafts- und Freundschaftsverbünde der Frauen in der Familie, durch Lehrer, Kantoren oder Rabbiner mit einem weiten Netzwerk oder durch professionelle Vermittler, sogenannte „Schadchen“, gesucht (Kaplan 1997: 134; Maurer 1991: 345). Die Zeitung als weiteres Medium der Vermittlung ließ sich in diese Tradition der Ehepartnersuche leicht einfügen. Mit ihr konnte der Radius der bestehenden Netzwerke vergrößert werden. Da 
auch in den beiden Annoncen, die in diesem Unterkapitel analysiert werden sollen, die jüdische Religion genannt wurde, ist es nicht außergewöhnlich, dass sie nicht von den Betreffenden selbst, sondern von nicht näher definierten Mittelspersonen geschaltet wurden.

Die erste Anzeige stammt aus der überregional erschienenen Nationalzeitung und trägt die durch Fettdruck und große Schrift hervorgehobene Überschrift „Ernstes Heiratsgesuch für eine Dame!“7 In der ersten Zeile des Textes wird die Suchende als ,israel[itisches], gebildetes junges Mädchen“ beschrieben, es folgt die Beschreibung der Herkunft ,aus guter und wohlhabender Familie“ und der durch Kommas abgetrennte Einschub ,welches einen kleinen äußerlichen Fehler im Gesicht hat". Hieran schließt sich die Beschreibung des Wunschpartners an, der ebenfalls der jüdischen Religion angehören und ihren Verhältnissen angemessen sein sollte, ,verwachsen, einen Krüppel oder ebenfalls mit einem Fehler behaftet, da sich die junge Dame nur so zu verheirathen wünscht, um eine glückliche und ruhige Häuslichkeit zu führen". Ähnlich wie bei der Annonce des Fabrikanten aus Wien ist anzunehmen, dass der Wunsch nach einem körperlich ähnlichen Partner die Wahl eines überregionalen, raumüberwindenden Mediums zur Eheanbahnung begünstigt hat.

Die zweite Annonce scheint fast das Gegenstück zu eben jenem, oben beschriebenen, taubstummen Fabrikanten zu sein. Sie wurde nur eine Woche nach seiner Anzeige, ebenfalls im Berliner Tageblatt geschaltet und trägt die ähnliche Überschrift „An Taubstumme“. ${ }^{8}$ Der Text beginnt mit der Angabe des Alters und der Religion der Suchenden und der Beschreibung des Wunschpartners als ,in guten Verhältnissen“ befindlich. Dies ist im Text die einzige Forderung an den künftigen Ehepartner, hier hat die Überschrift die Zielgruppe auf den ersten Blick zugeschnitten. Der weitere Text widmet sich wieder der Beschreibung der Suchenden. Diese sei „hoch gebildet“, spreche und schreibe fließend deutsch und englisch „,derart, daß man sie kaum für taubst[umm] hält“", wobei ersteres mit einer Klammer („Lippenspr[ache]“) versehen wurde. Des Weiteren wird die Intelligenz und das Aussehen der jungen Frau gelobt, die ,im Hausstand bewandert und liebenswürdigen Charakters" sei und „die ihrem Mann eine treue Gefährtin und Stütze sein würde“.

7 Nationalzeitung vom 05.04.1873, Chiffre E.B.600.

8 Berliner Tageblatt und Handelszeitung vom 22.07.1898, Chiffre K.J. 2490. 
Die letzten Angaben umreißen die Aufgabengebiete einer bürgerlichen Ehefrau im Kaiserreich. Während der Mann als Ernährer der Familie für den materiellen Unterhalt zuständig war, lag die häusliche Sphäre im Verantwortungsbereich der Frau. Dies bedeutete jedoch nicht, dass ihre Arbeit im Hauswesen und der Kindererziehung von ökonomischen Bedeutungen frei war. Die Frauen waren die sichtbaren Repräsentantinnen, sowohl des gegenwärtigen als auch des angestrebten sozioökonomischen Status ihres Ehemannes (Garland-Thomson 1997: 90). [MUTTERSCHAFT] Gisela Mettele stellt in ihrem Aufsatz über den privaten Raum als öffentlichen Ort der Geselligkeit heraus, dass der berufliche Erfolg des Mannes weniger als individuelle Leistung, denn als Familienleistung galt, an dem die häuslichen und repräsentativen Tätigkeiten ihren Anteil hatten (Mettele 1996: 162). Diese Auffassung setzte die Wirkungskreise der beiden Ehepartner komplementär zueinander als gemeinsame Arbeit am gesellschaftlichen Aufstieg. Durch die Einladung von Kollegen und Geschäftspartnern in die gut ausgestatteten und wohl geordneten Privaträume wurde der Eindruck wirtschaftlichen Wohlhabens nach außen getragen und ein bürgerliches Selbstverständnis gepflegt (Mettele 1996: 162, 168). Mit ihren verschiedenen Rollen der Ehefrau und Mutter, Managerin des Hauswesens und aktive Repräsentantin des Status gehörte der Aufbau eines bürgerlichen Lebensstils zu den integralen Aufgaben der Frau (Blumin: 1989: 191). Obwohl das Bild als „Engel des Hauses“ die Frau in ein spirituelles, vom Körper losgelöstes Spektrum setzte, war für die substantiellen Aufgaben im Hauswesen und der Repräsentation ein tätiger und den Schönheitsnormen entsprechender Körper unerlässlich (zu diesem Spannungsfeld vgl.: Dykstra 2001: 117).

Einen solchen kann die Suchende der Annonce der Nationalzeitung nicht bieten. Obwohl der Autor der Annonce ihren ,äußerlichen Fehler“ durch das vorangestellte Adjektiv „,klein“ abschwächte, scheint sich die Suchende dieses Umstandes bewusst gewesen zu sein. Sie suchte ausdrücklich einen Mann, der selbst Zeichen von Imperfektion trug, da für die Suchende, so die Erklärung in der Anzeige, dieser Aspekt die Vorbedingung für den Aufbau eines privaten Glücks darstellte. [VISUALISIERUNG] Während der Großkaufmann mit „Gesichtsfehler“ die Forderung nach einer schönen Partnerin über die nach materiellen Wohlstand stellt und der Eindruck entsteht, dass eine Repräsentantin, die durch körperliche Makellosigkeit und künstlerische Talente seine ökonomischen Erfolge unterstreichen konnte, gesucht wurde, 
koppelte die Suchende dieser Annonce eine glückliche Ehe an die körperliche Ähnlichkeit. Auf diese Weise wäre keiner der Partner in der Situation, die eigene Imperfektion als abträglich für den jeweils anderen abwägen zu müssen.

Auch die taubstumme Dame aus der Annonce des Berliner Tageblattes suchte, wie auch der taubstumme Fabrikant, einen in dieser Hinsicht gleichen Partner. Hier standen eventuell Überlegungen für eine gesicherte gegenseitige Kommunikation im Zentrum, wenn beide Partner die Gebärdensprache und andere Kommunikationsstrategien beherrschten. Trotz des Wunsches nach einem ebenfalls taubstummen Partner wurde jedoch auch in dieser Annonce Formulierungen genutzt, die auf einen Ausgleich vermeintlicher Einschränkungen durch die Taubstummheit abzielten. So wird die Bildung und Intelligenz der Suchenden betont, die ihr zu Kommunikationstechniken verhelfe, die dazu führen würden, „daß man sie kaum für taubst[umm] hält.“ Damit wurde dem Bild des inferioren Behinderten, dessen körperliche Abweichung eine Unzulänglichkeit in geistiger Hinsicht mit sich führe, sofort widersprochen und gleichzeitig dem Bildungsideal der jüdischen Religionsgruppe entsprochen. Dazu kommen die Schilderungen eines angenehmen Charakters und schönen Äußeren, sodass das Bild einer Frau aufgebaut wird, die sich sowohl nützlich machen kann, ${ }^{9}$ als auch alle Attribute sichtbarer häuslicher und repräsentativer Weiblichkeit besitzt.

\section{EXKURS: 100.000 M. MITGIFT ABER KLEINER KÖRPERLICHER FEHLER}

Die Überschrift dieses Exkurses ist gleichlautend mit einer Kapitelüberschrift aus dem Buch Die Heiratsannonce. Studien und Briefe, das 1908 von einem Joachim Werner herausgegeben wurde. Der Herausgeber schrieb nach eigener Angabe die Verfasser von Heiratsannoncen mit der Bitte an, ihm die eingegangen Antwortbriefe zukommen zu lassen und diese abdrucken zu

9 Heiratsannoncen jüdischer Frauen gingen häufiger als Annoncen nicht jüdischer Frauen auf Fähigkeiten wie Buchhaltung und Korrespondenz ein, die eine Mitarbeit in einem kaufmännischen Geschäft möglich machen. Dies ist neben dem Bildungsideal dieser Religionsgruppe evtl. auch auf die durch Familienunternehmen geprägte Gewerbestruktur dieser Gruppe zurückzuführen. 
dürfen (Werner 1908: 17). Dass es sich bei den Briefen um vom Autor erfundene Antwortschreiben handelt, erscheint nicht wahrscheinlich, jedoch kann nicht nachgewiesen werden, ob es sich um authentische Annoncen handelt oder ob diese von Werner selbst inseriert wurden, um so an vielfältiges Antwortmaterial zu kommen. Trotzdem soll hier auf einige der Antwortschreiben eingegangen werden, die auf eine besondere Heiratsannonce eingesandt wurden. In dieser Anzeige wurde ein Ehepartner für eine junge Waise mit 100.000 Mark Mitgift gesucht, die als musikalisch, liebenswürdig, mit sympathischem Äußeren, ,,aber mit kleinem körperlichen Fehler“ beschrieben wurde (Werner 1908: 79).

Nicht alle Reflektanten gingen auf den „körperlichen Fehler“ der beschriebenen Frau ein, auch nicht alle auf die hohe Mitgift. Ein Reflektant zog eine Verbindung zwischen den beiden Elementen, indem er in seinem Brief die Annahme zum Ausdruck brachte, dass die Verfasserin der Anzeige eine Tante der jungen Frau sei, die es bedauern würde, dass ein ,liebenswertes Wesen wegen eines kleinen körperlichen Fehlers vernachlässigt" würde (Werner 1908: 92). Er fasste damit die hohe Mitgiftsumme als Ausgleich für eine körperliche Imperfektion auf. Eine Interpretation, die viele seiner Mitbewerber geteilt haben könnten. Dass es ihnen nicht um diesen „Ausgleich“ ging, versuchten viele von ihnen durch Erklärungen über hohe moralische Grundsätze, eigenes Vermögen oder die geringe Relevanz von materiellen Abwägungen bei der Partnerwahl hervorzuheben (Werner 1908: 90/98). Indem er auf das körperliche Beschreibungselement einging, verband ein Inserent diese Elemente und legte dar, dass aus seiner Sicht ,solche Damen das beste Gemüt und den besten Charakter" hätten und es diese Eigenschaften seien, die er suche (Werner 1908: 87). Hier liegt ein Narrativ vor, dass Lennard Davis als Sentimentalisierung von Behinderung im Bürgertum wertet (Davis 1995: 4). Dabei wird eine Geschichte mit einem Helden oder einem Opfer entworfen, dem ein Set von positiven Attributen zugeschrieben wird (Davis 1995: 4), wie ein edles Wesen oder ein heldenhafter Zug (Davis 1995: 106). Im oben beschriebenen Fall wäre es die Zuschreibung eines besonders tugendhaften Wesens, das sich mit dem bereits genannten spirituellen Bild der engelhaften, ätherischen und damit körperbefreiten Weiblichkeit deckte. Davis versteht dieses Vorgehen des Bürgertums als ,,attempt to erase the difference by dressing it in moral raiment" (Davis 1995: 106). Der Reflektant 
füllt die Leerstellen, die die Beschreibung der elf Zeilen lange Anzeige hinterlassen hat so aus, dass ein für ihn kohärentes Bild der Suchenden entsteht. Damit stülpt er jedoch der ihm unbekannten Person eine ganze Identität über.

Einer weiteren Strategie, um die wahrgenommene Differenz zu tilgen, bedienten sich mehrere Bewerber, die von eigenen körperlichen Beeinträchtigungen oder denen von Familienmitgliedern schrieben (Werner 1908: 86/107). Sie drückten damit Vertrautheit und Verständnis aus und konnten gleichzeitig ein Argument dafür liefern, dass es nicht allein die hohe Mitgift sei, die sie zu einer Antwort auf die Annonce bewogen hatte. Ein Inserent drückte sogar die Hoffnung aus, durch ,schöne Ausflüge [...] mit Gottes Hilfe Ihren körperlichen Fehler zu heilen" (Werner 1908: 103) und bewegte sich damit, wiederum ohne nähere Informationen über die betreffende Person und den „kleinen körperlichen Fehler“ zu haben, im gängigen Defizitnarrativ, in dem Behinderung in jedem möglichen Fall geheilt werden sollte (Garland-Thomson 2003: 421), um eine Normalisierung anzustreben.

\section{ZUSAMMENFÜHRUNG}

„Feminization increases a woman's cultural capital; disability reduces it“ (Garland-Thomson 1997: 28) - diese Beurteilung von Rosemarie GarlandThomson schlägt die Brücke zwischen dem, was in der Einleitung über den Einsatz von Kapitalsorten auf dem Heiratsmarkt ausgeführt wurde zu den Inhalten der vorgestellten Heiratsannoncen.

In Heiratsannoncen müssen sich die Suchenden auf begrenztem Platz möglichst positiv darstellen, um von potentiellen Heiratskandidaten als ,gute Partie" wahrgenommen zu werden. Materieller Wohlstand, Bildung und die Herkunft aus einer guten Familie konnten dabei ebenso als Kapitalsorten eingesetzt werden wie ein attraktives Äußeres. Die Ausführungen im Hauptteil haben dargelegt, dass eine als Behinderung eingestufte Abweichung von der Norm dagegen als Defizit wahrgenommen wurde, die die soziale Positionierung der Betreffenden negativ beeinflusste. Dass sich die Inserierenden des Quellenkorpus trotzdem mit einem in der Gesellschaft als Makel angesehenen Attribut in einem öffentlichen Kommunikationsraum wie der Heiratsannonce vorstellten, war deshalb selten. Im Gegenteil ist es denkbar, dass andere Inserierende in dieser ersten Kontaktaufnahme über die Zeitung Imperfektionen, seien es körperliche oder soziale (z.B. ein uneheliches Kind, eine 
Scheidung), zunächst verschwiegen, um einen durchweg positiven ersten Eindruck zu wecken und ihre Chancen nicht bereits zu Beginn des Prozesses zu schmälern.

Doch auch den Verfasser_innen der Anzeigen in diesem Quellenkorpus war es ein Anliegen, ein positives Bild von sich selbst zu zeichnen, das sie von den gesellschaftlichen Schreckensbildern, abhängiger, devianter, inferiorer und behinderter Menschen abheben konnte. Dafür nutzten sie nicht nur textuelle Anordnungen, die der als körperliche Abweichung wahrgenommenen Beschreibung eine untergeordnete Rolle verliehen oder Formulierungen, die diese abschwächten, sondern bauten bewusst das Bild gelungener Weiblichkeit bzw. Männlichkeit auf. Mit den Kapitalangaben einer selbstständigen Beschäftigung und geordneten Verhältnissen machten die männlichen Inserenten deutlich, dass sie der Rolle des Ernährers der Familie voll und ganz gerecht werden konnten. Auch die weiblichen Suchenden wurden durch ihre Mittelspersonen im Spektrum einer gelungenen Geschlechtsidentität beschrieben, indem Attribute wie Bildung, Intelligenz, Sozialisation in einer angesehenen Familie, haushälterische Fähigkeiten, ein liebenswerter Charakter und, im Falle der taubstummen jungen Frau aus dem Berliner Tageblatt, auch äußerliche Schönheit hervorgehoben wurden, die als Kapitalsorten für die Rolle der „Herrin des Hauses“ eingesetzt werden konnten. Auch hier bestimmte also ein deutliches „doing gender“ die Selbstbeschreibung der Annoncen.

Mit diesem auf Leistung abzielenden ,,doing gender" stellten die Autoren sowohl für die Männer als auch für die Frauen eine deutliche Negation der gesellschaftlichen Defizitzuschreibungen her, die sich durch die abschwächenden Formulierungen, die in direktem Bezug zur körperlichen „Andersartigkeit“" standen, wie dass diese ,nicht im geringsten“ entstelle oder beruflich hindere bzw. dass sie durch gekonnte Fertigkeiten so ausgeglichen werde, dass man die Betreffende „,kaum für taubst[umm] hält“, zu einem „doing ability“ verbindet.

Zuletzt soll noch ein Punkt in die Überlegungen mit aufgenommen werden, der sich nicht auf den allgemeinen Heiratsmarkt bezieht, sondern eine Implikation des speziellen Kommunikationsraumes Heiratsannonce ist. Auf den einleitenden Seiten wurde bereits erwähnt, dass die Eheanbahnung durch Annonce im Kaiserreich hoch umstritten war und Heiratsanzeigen als „Horte der Unzucht“" wahrgenommen wurden. Das Bürgertum und die aufstiegsorientierte Mittelschicht sahen ihre Hochkultur durch eine abweichende Kultur 
der unteren Klassen bedrängt, die sie als primitive „Populärkultur“ ablehnten. Wirtschaftsunternehmen wie die neuen Massenmedien, zu denen auch das Berliner Tageblatt, die Berliner Zeitung und die Münchner neueste Nachrichten zählten, die diese Populärkultur durch eine stärker boulevardisierte Form zu tragen schienen, statt auch den unteren Schichten die bürgerlichen Verhaltens- und Bildungswerte näher zu bringen, wurden nicht als Kulturträger anerkannt und abgelehnt. Der schlechte öffentliche Ruf der Trägermedien als Quellen der Kulturerosion und Ursachen der zunehmenden „Nervosität der Zeit“ (Radkau 1998: 224f.) fiel auch auf die Heiratsannonce, zeichneten sich doch gerade die kritisierten Massenzeitungen dadurch aus, dass sie täglich mehrere Heiratsgesuche abdruckten und diese zudem in unmittelbarer Nähe zu Anzeigen von Hebammen, „diskreten Geburten“ und Verhütungsmethoden platziert waren. Eine Verbindung der Inserenten von Heiratsannonce zu den als unmoralisch und degenerativ gefürchtetem Mitgliedern der unteren Schichten war da leicht gezogen. Brendan Gleesons Ausführungen über die industrialisierte Stadt legen nahe, dass durch die erhöhte Sichtbarkeit behinderter Menschen im öffentlichen Raum, die durch ihre dort ausgeführten Gewerbe als Angehörige der gesellschaftlichen Randgruppen kategorisiert wurden, eine allgemeine Einordnung zu der Gruppe suspekter, devianter oder pathologisch eingeschätzter Personen erfolgte (Gleeson 1999: 110/124). Mit der Darstellung von ökonomischem Erfolg und einem Selbst, das den bürgerlichen Geschlechterpositionen entsprach, wollten die Inserierenden wahrscheinlich auch eine Einordnung ihrer Selbst in den Kreis dieser unterbürgerlichen Schicht entgegenwirken und stattdessen ihre Zugehörigkeit zur bürgerlichen Schicht betonen.

Führt man nun alle ausgeführten Punkte zusammen, können die sprachlichen Handlungen, die in den hier vorgestellten Heiratsannoncen vorgenommen wurden, als ein komplexes, sich gegenseitig bedingendes ,doing class while doing ability while doing gender" interpretiert werden. [EINLEITUNG] Diese reziproke Kette macht deutlich, wie vielen verschiedenen Anforderungen, wie vielen verschiedenen Identitätszuschreibungen und -verweigerungen sich Menschen mit körperlichen Besonderheiten auf dem Heiratsmarkt gegenübersahen und wie sie sich in den Diskurs um die Implikationen körperlicher „Andersheit“ im medialen Kommunikationsraum der Heiratsannoncen einschrieben. 


\section{LITERATUR}

Berghaus, Margot (1986): Der Auftakt persönlicher Beziehungen. „Besonderheiten" bei Kontakt und Kommunikation durch Heirats- und Bekanntschaftsanzeigen, in: Zeitschrift für Soziologie 15, S. 56-67.

Bernstein, Eduard (1895): „Die moderne Ehe und die Heiratsannonce“, in: Die Neue Zeit. Wochenschrift der deutschen Sozialdemokratie (Heft 27), S. 16-21.

Bloch, Iwan (1908): Das Sexualleben unserer Zeit in seiner Beziehung zur modernen Kultur, Berlin: Marcus, S. 786-791.

Blumin, Stuart M. (1989): The emergence of the middle class. Social experience in the American city 1760-1900, Cambridge: Cambridge University Press.

Bösl, Elisabeth (2010): Was ist Disability History? Zur Geschichte und Historiographie von Behinderung, in: Dies./Anne Klein/Anne Waldschmidt (Hg.), Disability History. Konstruktionen von Behinderung in der Geschichte. Eine Einführung, Bielefeld: transcript, S. 29-43.

Borsay, Anne (2006): Industrialization, in: Albrecht, Gary L. (Hg.), Encyclopedia of Disability, Thousand Oaks: Sage Publications, S. 947-950.

Bourdieu, Pierre (1987): Das symbolische Kapital, in: Ders., Sozialer Sinn. Kritik der theoretischen Vernunft, Frankfurt a. M.: Suhrkamp, S. 205222.

Bourdieu, Pierre (1983): Ökonomisches Kapital, kulturelles Kapital, soziales Kapital, in: Reinhard Kreckel (Hg.), Soziale Ungleichheiten, Göttingen: Schwartz, S. 183-198.

Coontz, Stephanie (2005): Marriage. A History. From Obedience to Intimacy or how love conquered marriage, New York: Viking.

Davis, Lennard J. (1997): Constructing Normalcy. The Bell Curve, the Novel and the Invention of the Disabled Body in the Nineteenth Century, in: Ders. (Hg.), The Disability Studies Reader, London: Routledge, S. 9-28.

Davis, Lennard (1995): Enforcing Normalcy. Disability, deafness and the body, London/New York: Verso.

Dykstra, Natalie A. (2001): „Trying to be idle.“ Work and Disability in the Diary of Alice James, in: Paul K. Longmore/Lauri Umansky (Hg.), The New Disability History. American Perspectives, New York: New York University Press, S. 107-130. 
Frey, Tamara (2017): „Strengste Verschwiegenheit auf Manneswort.“ Eine Analyse von Heiratsannoncen im Kaiserreich (eDissertation Universität Göttingen).

Garland-Thomson, Rosemarie (2003): Andere Geschichten, in: Petra Lutz, Thomas Macho, Gisela Staupe, Heike Zirden (Hg.), Der (im-)perfekte Mensch. Metamorphosen von Normalität und Abweichung, Köln: Böhlau, S. 419-425.

Garland-Thomson, Rosemarie (1997): Extraordinary bodies. Figuring physical disability in American culture and literature, New York: Columbia University Press.

Gleeson, Brendan (1999): Geographies of Disability, New York: Routledge. Kaplan, Marion (1997): Jüdisches Bürgertum. Frau, Familie und Identität im Kaiserreich, Hamburg: Dölling und Galitz.

Kaschuba, Wolfgang (1990): Lebenswelt und Kultur der unterbürgerlichen Schichten im 19. und 20. Jahrhundert, München: Oldenbourg Wissenschaftsverlag.

Kraus, Elisabeth (1999): Rudolf Mosse, Vom Werbekönig zum Pressezaren, in: Günther Schulz (Hg.), Geschäft mit Wort und Meinung. Medienunternehmer seit dem 18. Jahrhundert. Bündiger Forschungen zur Sozialgeschichte, München: Boldt im Oldenbourg Verlag, S. 77-101.

Lutz, Petra/Macho, Thomas/Staupe, Gisela/Zirden, Heike (2003): Einleitung der Herausgeber, in: Dies. (Hg.), Der (im-)perfekte Mensch. Metamorphosen von Normalität und Abweichung, Köln: Böhlau, S. 10-17.

Manderscheidt, Katharine (2008): Pierre Bourdieu. Ein ungleichheitstheoretischer Zugang zur Sozialraumforschung, in: Fabian Kessl/Christian Reutlinger (Hg.), Schlüsselwerke der Sozialraumforschung. Traditionslinien in Texten und Kontexten, Wiesbaden: VS, S. 155-168.

Maurer, Trude (1991): Partnersuche und Lebensplanung. Heiratsannoncen als Quelle für die Sozial- und Mentalitätsgeschichte der Juden in Deutschland, in: Peter Freimark/Alice Jankowski/Ina Lorenz (Hg.), Juden in Deutschland. Emanzipation, Integration, Verfolgung, Vernichtung, Hamburg: Christians, S. 344-375.

Mettele, Gisela (1996): Der private Raum als öffentlicher Ort. Geselligkeit im bürgerlichen Haus, in: Dieter Hein/Andreas Schultz (Hg.), Bürgerkultur im 19. Jahrhundert. Bildung, Kunst und Lebenswelt, München: Beck, S. 155-170. 
Raab, Heike (2007): Intersektionalität in den Disability Studies. Zur Interdependenz von Behinderung, Heteronormativität und Geschlecht, in: Anne Waldschmidt/Werner Schneider (Hg.), Disability Studies. Kultursoziologie und Soziologie der Behinderung. Erkundungen in einem neuen Forschungsfeld, Bielefeld: transcript, S. 127-148.

Radkau, Joachim (1998): Das Zeitalter der Nervosität. Deutschland zwischen Bismarck und Hitler, Darmstadt: Wissenschaftliche Buchgesellschaft.

Schroer, Markus (2012): Räume, Orte, Grenzen. Auf dem Weg zu einer Soziologie des Raums, Frankfurt a. M.: Suhrkamp.

Waldschmidt, Anne (2010): Warum und wozu brauchen die Disability Studies die Disability History? in: Dies./Elisabeth Bösl/Anne Klein (Hg.), Disability History. Konstruktionen von Behinderung in der Geschichte. Eine Einführung, Bielefeld: transcript, S. 13-27.

Werner, Joachim (1908): Die Heiratsannonce. Studien und Briefe, Berlin: Aronhold.

West, Candace/Don H. Zimmerman (1991): Doing gender, in: Judith Lorber/Susan A. Farrell (Hg.), The social construction of gender, Newbury Park: Sage Publications, S. 13-37.

Zwahr, Hartmut (1978): Konstituierung des Proletariats als Klasse. Strukturuntersuchungen über das Leipziger Proletariat während der Industriellen Revolution, Berlin: Akademie-Verlag. 
